\title{
Mediatisierte sexualisierte Gewalt gegen Kinder und Jugendliche
}

Verbreitete Vorstellungen von mediatisierter sexualisierter Gewalt werden durch Diskussionen über Cybergrooming (sexuelle Ausbeutung mittels digitaler Medien) durch Fremdtäter*innen bzw. die Verbreitung und den Konsum von Missbrauchsabbildungen (Kinderpornografie) beherrscht (Vobbe \& Kärgel, im Druck). Tatsächlich werden digitale Medien jedoch auch von Gewaltausübenden des sozialen Nahraums (z. B. Familienmitglieder, Mitarbeitende pädagogischer Einrichtungen) täter*innenstrategisch genutzt. Wir definieren in Erweiterung des Gewaltverständnisses nach Hamby (2017) mediatisierte sexualisierte Gewalt als a) Verletzungshandlungen, mit denen b) gewaltausübende Personen absichtsvoll ${ }^{1}$ eigene Bedürfnisse c) gegen die sexuelle Selbstbestimmung und/oder das Einvernehmen von Kindern und Jugendlichen durchsetzen und d) digitale Medien Instrument (z. B. Anbahnung über Messaging-Dienste) oder Kontext (z. B. Tatort im Falle der Verbreitung von Nacktaufnahmen) der Handlungen sind. Die Formen mediatisierter sexualisierter Gewalt sind demnach vielgestaltig:

- sexualisierte Ansprache von Kindern oder nicht einvernehmliche sexualisierte Ansprache Jugendlicher über digitale Medien (z. B. soziale Netzwerke, Online-Spiele, Messaging-Dienste),

- Aufforderung, sexualisierte Foto- oder Videoaufnahmen oder Nacktaufnahmen herzustellen, sich selbst (vor der Kamera) zu befriedigen oder Dritte in sexualisierte Handlungen zu verwickeln,

\footnotetext{
${ }^{1}$ Als „Absicht“ verstehen wir in einem devianztheoretischen Sinne, wenn Menschen den Nutzen einer Handlung als höher einschätzen als Widerstände, die dem Verhalten entgegenstehen könnten. Das bedeutet nicht notwendigerweise eine vollumfängliche Einsicht, Reflexion oder Abwägung der Handlungsziele (Dollinger \& Raithel, 2006). Sie markiert aber den Unterschied zu einer versehentlichen Grenzverletzung.
} 
- kommerzielle sowie nicht kommerzielle Herstellung und Verbreitung von Missbrauchsabbildungen,

- Einbettung sexuellen Missbrauchs - auch innerfamiliär, institutionell oder in anderen Abhängigkeitsverhältnissen - in digitale Kommunikation, Schweigegebote und Drohungen,

- digitale Überwachung Gewaltbetroffener (z. B. Kommunikations- und Bewegungskontrolle) zwecks Aufbau von Druck und/oder Sicherstellen der Geheimhaltung der sexualisierten Gewalt oder zur Herstellung des Gefühls totaler Kontrolle,

- Androhung der Veröffentlichung oder Verbreitung von sexualisierten Behauptungen und Chatprotokollen sowie Foto- und Videoaufnahmen, die sexualisiert sind oder als intim erlebt werden,

- sexualisierte Kommentierung von Foto- und Videoaufnahmen,

- Konsum von Missbrauchsabbildungen,

- Konfrontation von Kindern und Jugendlichen mit Pornografie oder Missbrauchsabbildungen,

- gemeinsamer Konsum von Pornografie oder Missbrauchsabbildungen mit Kindern und Jugendlichen; besonders in Verhältnissen, die durch Reifeunterschiede oder Abhängigkeiten (Familie, Lehrer*innen-Schüler*innenVerhältnisse) gekennzeichnet sind.

\subsection{Täter*innenstrategische Nutzung digitaler Medien}

Gewaltausübende ziehen aus kindlichen und jugendlichen Mediennutzungsgewohnheiten insoweit einen Vorteil, als sie ihre Absichten und Motivationen weder offenlegen noch rechtfertigen müssen. Das Teilen von Foto- und Videoaufnahmen stellt unter Jugendlichen schließlich eine Praxis der (sexuellen) Identitätsfindung dar (Döring, 2019; Matthiesen \& Dekker, 2018). Die Unterscheidung zwischen einer grenzachtenden und einer sexualisiert grenzverletzenden Interaktions- und Beziehungsgestaltung wird dadurch erschwert. Neben den eigenen Vorstellungen, die das Bild des Gegenübers maßgebend prägen (s. Kap. 1), trägt hierzu bei, dass Kindern und Jugendlichen suggeriert wird, dass eine konsensuelle Beteiligung an sexualisierter Interaktion auch bedeute, beispielsweise geäußerte Fantasien tatsächlich erfüllen zu müssen. Das Sprichwort „Wer A sagt, muss auch B sagen" beschreibt treffend die Situation, in der sich Kinder und Jugendliche an der Schwelle zwischen Grenzwahrung und Grenzüberschreitung befinden. Entscheidungsspielräume erscheinen plötzlich begrenzt. Ein „Nein“ könnte vom 
Gegenüber als inkonsequent und nicht authentisch wahrgenommen werden. Wenn Gewaltausübende auf eine etwaige Zögerlichkeit oder Verweigerung enttäuscht oder verletzt reagieren oder aber andeuten, das Interesse am weiteren Kontakt verloren $\mathrm{zu}$ haben, entsteht aufseiten von Kindern und Jugendlichen aus einem Zusammenspiel der Angst vor einem Beziehungsverlust und einer habitualisierten Sexualisierung von Kindheit und Jugend umso mehr der Eindruck, sie seien in der Pflicht, die Erwartungen des Gegenübers zu erfüllen. Die Wahrnehmung, sich aktiv beteiligt zu haben, ist dabei meist schambesetzt. Gewaltausübende instrumentalisieren dies insoweit, als die Möglichkeit einer Veröffentlichung von beispielsweise Chatverläufen, die die scheinbar aktive Beteiligung der Betroffenen ,belegen“, als Druckmittel eingesetzt wird (Broome et al., 2018; Whittle et al., 2014). Ein solches Machtungleichgewicht entsteht aber auch, wenn Gewaltausübende digitale Medien dazu nutzen, um Informationen zu manipulieren oder die eigenen Absichten gezielt vorzuenthalten. Wir sprechen in diesem Zusammenhang von Informationskontrolle. Dies geht über gängige Vorstellungen eines Verschleierns der eigenen Identität hinaus und kann beispielsweise im Vortäuschen romantisch-liebevoller Interessen Ausdruck finden. Dieserart wird das wechselseitige Vertrauen bestärkt und vermeintlich Verständnis für Bedenken, Sorgen und Probleme vorgegeben. Die Sogwirkung hiervon kann so stark sein, dass Kinder und Jugendliche sich von ihrer Peergruppe oder Herkunftsfamilie isolieren oder diese sogar verlassen. Gleichzeitig können die Weichen für eine Sexualisierung gestellt werden. Aufmerksamkeit und Zuneigung sind Schlüsselelemente der täter*innenstrategischen Beziehungsgestaltung. Das gilt sowohl für Personen, die die Kinder und Jugendlichen über digitale Medien kennenlern(t)en, wie auch für ihnen bereits bekannte Personen (z. B. Familienmitglieder, Freund*innen, Vereinskamerad*innen, Bekannte) (Vobbe \& Kärgel, im Druck). Als Geschenk getarnt werden mobile Endgeräte wie Smartphones oder Tablets nebstdem genutzt, um Vertrauen aufzubauen und Beziehungen $\mathrm{zu}$ intensivieren. Smartphones sind Statussymbole, die bei jungen Menschen Begeisterung auslösen. Gewaltausübende machen sich mit verschenkten Geräten beliebt, verlangen Gegenleistungen dafür und nutzen dieselben Medien zugleich, um Gewalt anzubahnen. Eingebettet in heteronormative Geschlechterordnungen ${ }^{2}$

\footnotetext{
${ }^{2}$ Heteronormativität „benennt Heterosexualität als Norm der Geschlechterverhältnisse“ (Wagenknecht, 2007, S. 17), die als solche Erwartungen an akzeptiertes und nicht akzeptiertes Verhalten schafft. So erzeugt sie vor dem Hintergrund eines binären Geschlechterverständnisses beispielsweise den Druck, ,sich selbst über eine geschlechtlich und sexuell bestimmte Identität zu definieren, wobei die Vielfalt möglicher Identitäten hierarchisch angeordnet ist und im Zentrum der Norm die kohärenten heterosexuellen Geschlechter Mann und Frau stehen." (ebd., S. 17).
} 
und hegemoniale Vorstellungen von Männlichkeit und Weiblichkeit ${ }^{3}$ versprechen mobile Endgeräte und damit auch gemeinsame sexualisierte Aktivitäten, wie zum Beispiel der gemeinsame Konsum pornografischer Inhalte oder das Aufnehmen sexualisierter Fotos, eine soziale Aufwertung durch exklusive und teils bündische Zugehörigkeit. Intime sowie verletzende Foto- und Videoaufnahmen oder gespeicherte Kommunikationsverläufe (z. B. Screenshots von Chatverläufen, E-Mails) führen ungeachtet dessen oftmals dazu, dass sexualisierte Gewalt fortgeführt und geheim gehalten wird - beispielsweise indem Gewaltbetroffenen angedroht wird, die Aufnahmen über digitale Medien zu verbreiten (Vobbe \& Kärgel, 2020). In einigen Fällen erfüllt die Kommunikation über digitale Medien den ausschließlichen Zweck, Gewaltbetroffene einschließlich ausgesprochener Schweigegebote zu kontrollieren.

\subsection{Mehrfachbetroffenheit und Folgebelastungen}

Über die Folgeerscheinungen mediatisierter sexualisierter Gewalt ist derzeit vergleichsweise wenig bekannt. Die existierende Fachliteratur geht jedoch bereits bei vorwiegend online verübter sexualisierter Gewalt von ähnlichen klinischen und psychosozialen Belastungsreaktionen - Symptome einer posttraumatischen Belastungsstörung, Depression, Entwicklungsstörung, Gefühle von Ohnmacht, Angst und Scham, Einsamkeit, Isolation sowie Selbstvorwürfe - aus wie bei körperlichen Übergriffen (vgl. Wells \& Mitchell, 2007; Dekker et al., 2016). Wir stellen unter Berücksichtigung unserer im Laufe der Zeit gewonnen Einsichten die Hypothese auf, dass eine Mediatisierung der sexualisierten Gewalt die Belastungen betroffener Kinder und Jugendlicher verstärkt. Schließlich erleben sie meist nicht nur einen mehrfachen Kontrollverlust, sondern sind darüber hinaus mehrfachbetroffen. Unseren empirischen Beobachtungen zufolge ist die Mehrfachbetroffenheit eine Konsequenz der charakteristischen Eigenschaften digitaler Medien bzw. des Einsatzes digitaler Medien. Über mobile Endgeräte kann sexualisierte Gewalt jederzeit und überall verübt werden (z. B. Aufforderung zu Nacktfotos, Konsum von Missbrauchsabbildungen). Sie kann jenseits des Einflusses Gewaltbetroffener unkontrollierbar aufgedeckt werden; etwa indem das Stigma des Opferseins in soziale Bezugssysteme Gewaltbetroffener, z. B. Familie oder Peergruppe, hineingespielt wird oder indem sexualisierte Foto- oder Videoaufnahmen über digitale

\footnotetext{
${ }^{3}$ Der Begriff der hegemonialen Männlichkeit geht auf die australische Männerforscherin Connell (2015) zurück und bezieht sich auf gesellschaftliche Praxen, die die sozial dominante Rolle des Mannes und die untergeordnete Rolle von Frauen und als statusniedrig gelabelten Männern (z. B. homosexuelle Männer) stärken (ebd.).
} 
Medien veröffentlicht werden. Ebenso kann es trotz entsprechender Interventionen immer wieder zu einem erneuten Kontakt mit der gewaltausübenden Person kommen. Demnach kann mediatisierte sexualisierte Gewalt als genuin raum- und zeitüberschreitend verstanden werden, weshalb wir in diesem Zusammenhang von einer Transzendierung mediatisierter sexualisierter Gewalt sprechen. Wenn es zu einer (wiederholten) Veröffentlichung oder Verbreitung von Missbrauchsabbildungen oder intimitätsverletzenden Fotos, dem Anstiften von Demütigung und Bloßstellung (z. B. Täter*innen-Opfer-Umkehr, Stigmatisierung der Opferrolle, soziale Ausgrenzung), einem Fortbestehen des Kontakts zur gewaltausübenden Person, einer unkontrollierbaren Aufdeckung o. Ä. kommt, werden Gewaltbetroffene von ihrer erlebten Gewalt wiedereingeholt. Mit der Mediatisierung der sexualisierten Gewalt - etwa durch das Verbreiten von Missbrauchsabbildungen oder sexualisierter Foto- oder Videoaufnahmen - wird somit nicht selten ein Gewaltkreislauf angestoßen, der Gewaltbetroffene von der ersten Gewaltgeschichte (z. B. filmisch dokumentierte sexualisierte Misshandlung) in weitere Gewaltgeschichten (z. B. Verbreitung der Videoaufnahmen in der Peergruppe einschließlich anschließender Diskreditierung) führt. Für eine Mehrfachbetroffenheit ist die Wiedereinholung allerdings weniger entscheidend, als vielmehr das Bewusstsein für das prinzipielle Risiko. Dieses löst allgegenwärtige und andauernde Ängste aus. So schildern betroffene Kinder und Jugendliche beispielsweise, dass sie bestimmte soziale Netzwerke aus Angst vor einem Täter*innen-Kontakt nicht mehr nutzen oder dass sie häufiger in digitalen Medien recherchieren, um herauszufinden, ob Abbildungen von ihnen in sozialen Netzwerken kursieren. Die Angst wurzelt also maßgeblich in wahrgenommenen Ungewissheiten und Unbestimmbarkeiten. Werde ich der gewaltausübenden Person zukünftig in digitalen Medien begegnen? Wird sie mir abermals Gewalt antun? Werden meine Nacktfotos veröffentlicht oder verbreitet? Es sind Fragen wie diese, die Gewaltbetroffene teils noch Jahre nach den Gewaltwiderfahrnissen begleiten. Die mediatisierte sexualisierte Gewalt wird infolge als diffus, sprich uneindeutig, schatten- und schemenhaft, erlebt. Durch das Empfinden von Diffusion und Angst setzt sich die sexualisierte Gewalt auf einer leiblichen sowie kognitiv-affektiven Ebene des Erlebens Gewaltbetroffener fort - um nicht zu sagen, sie transzendiert sich hinsichtlich ihrer Folgeerscheinungen (Kärgel \& Vobbe, 2020). 


\subsection{Stereotype, Wahrnehmungsblockaden und Verdeckungszusammenhänge}

In öffentlichen und wissenschaftlichen Debatten über die mediatisierte Sexualentwicklung und Beziehungsgestaltung junger Menschen dominieren einerseits Positionen, die Risiken fokussieren. Andererseits fallen Beiträge auf, die besonders die Weiterentwicklung jugendlicher Medien- und Sexualkompetenzen hervorheben. In beiden Fällen wird nahezu ausschließlich das Sextingverhalten von Kindern und Jugendlichen, also der Austausch von sexualisierten oder sexuellen Fotos, Videos und Nachrichten (Rice \& Watson, 2016; Machimbarrena et al., 2018; Gámez-Gaduix \& Mateos-Pérez, 2019), in den Blick genommen. Grundsätzlich wird dieses vermeintlich kollektive Medienhandeln junger Menschen tendenziös und emotionalisiert diskutiert (Dekker \& Koops, 2017). Eine mediatisierte Beziehungsgestaltung geht nicht automatisch mit einem Zugewinn an sozialen Befähigungen einher, schließlich zielen Medien- und Sexualpädagogik nicht grundlos auf deren Förderung ab. Jedoch ist das mediatisierte Beziehungsverhalten Jugendlicher vielfältiger, als dass es auf Sexting beschränkt werden könnte. Zumal Sexting zwar Risiken birgt, diese sich jedoch insbesondere in einem Missbrauch von Vertrauen und nicht primär dem Verhalten der Betroffenen begründen. Sexting kann demgegenüber auch als gegenseitige, selbstbestimmte Praktik und damit als Versuch der Selbstermächtigung junger Menschen verstanden werden (Gámez-Gaduix \& Mateos-Pérez, 2019). Die Beziehungsgestaltung von Kindern und Jugendlichen auf Sexting und Sexting auf eine risikobehaftete Interaktionsform zu reduzieren, führt zu einem verengten Bild von kindlichem und jugendlichem Medienhandeln. Im Zusammenhang des HUMANProjekts wurden wir immer wieder mit vergleichbaren Stereotypen konfrontiert. Veranschaulichend ein Zitat auf einem Interview mit einer Sozialarbeiterin:

IP: „Nein, auch man muss auch mit dem Handy keine Pornos drehen, man muss keine sexuellen Handlungen filmen, man muss sowas nicht filmen mit dem Handy. Alle Medien, wo man was verschicken konnte, exen, sofort. Ist natürlich idiotisch, das gibt es nicht so."

I: „Es ist schön. Hört sich schön an.“

IP: „Es wäre so eine heilere Welt.“

I: „Ja.“

IP: „Eine heilere Welt.“

Interview, Schulsozialarbeiterin 
Die Äußerung der Sozialarbeiterin wie auch die Reaktionen der interviewführenden Person sind in ihrem Zusammenhang zu betrachten, nämlich der Verhandlung eines konkreten Falls mediatisierter sexualisierter Gewalt. In ihr drückt sich eine situative Hilflosigkeit aus, die nicht zwingend auf eine grundlegende Haltung der Sprechenden schließen lässt. Im erwähnten Gegensatz zwischen digitalen Medien (,allen Medien") und einer Technik, mit der man alle Medien, die sexuelle Handlungen zeigen, vernichten wollte (,exen“), wird diese Ohnmacht besonders deutlich. Nicht minder argumentiert die Sozialarbeiterin, dass „man“ - gemeint sind Jugendliche - „keine Pornos“ drehen müsse. Im konkreten Fall hat dies ein gewaltbetroffenes Mädchen auf Aufforderung getan. Die Sozialarbeiterin stellt somit einen Zusammenhang zwischen Gewaltbetroffenheit im Allgemeinen, verallgemeinerten Ansichten zum Medienhandeln Jugendlicher und dem betroffenen Mädchen her. Die Argumentation weist Berührungspunkte zu Vergewaltigungsund Opfermythen auf. Solche kennzeichnet, dass die Verantwortung für Gewalt nicht bei den Gewaltausübenden, sondern bei gesellschaftlichen Umständen (z. B. sexuelle Verwahrlosung der Jugend, Sexting, Gewalt geht von „Ausländern“ aus) oder bei Betroffenen selbst gesucht wird (z. B. kurzer Rock, sexuelle Freizügigkeit) (Goh et al., 2021). Stereotype Vorstellungen von Jugendkultur, die Gewaltund Opfermythen hervorbringen, erschweren Prävention und Intervention, weil sie den Blick auf die Lebenssituation junger Menschen und die Bedürfnisse Betroffener verstellen. Sie verkomplizieren gegenseitiges Verstehen zwischen Helfenden und Adressat*innen. Teils fördern sie sogenanntes Victim-Blaming oder Slut-Shaming. Damit sind weitere Abwertungen Betroffener als „Schlampe“, „Außenseiter“, „ewiges Opfer“ oder im homofeindlichen Sinne „schwul“ gemeint. Sie sind außerdem eine Wahrnehmungsblockade für die Gewalt und erschweren deren Aufdeckung.

In den Kommunikationswissenschaften wird von digitalen Medien als Strukturverstärkern gesprochen. Damit ist gemeint, dass soziale Zusammenhänge, Gegensätze und sozialer Status digital stärker hervortreten (Valkenburg et al., 2006). Im Online-Offline-Kontinuum spiegeln somit auch gesellschaftliche Rollenbilder und -erwartungen wider, welche über Teilhabe, Diskriminierung, Ressourcen und Befähigung mitentscheiden. Dabei ist das Konzept einer Strukturverstärkung nicht linear zu verstehen. Paradoxerweise kann es bedeuten, dass digitale Medien aufgrund gesellschaftlicher Benachteiligung als besonders teilhabenotwendig erlebt werden, da sie den Zugang zu bestimmten Lebensbereichen vereinfachen oder erst erlauben. Exemplarisch sei darauf verwiesen, welche Bedeutung Smartphones für geflüchtete Menschen haben, um beispielsweise die 
Fluchtroute zu planen, zu koordinieren, mit der Familie bzw. dem sozialen Umfeld in Kontakt zu bleiben oder sich im Ankunftsland zu vernetzen und zu orientieren (Alencar et al., 2019). Für Menschen mit Behinderung stellen digitale Medien ein Instrument sexueller Teilhabe und Communion jenseits gesellschaftlicher Zuschreibungen und Tabuisierung dar (Hall, 2018). Aus ähnlichen Gründen sind digitale Medien beim Coming-out von trans- und nicht heterosexuellen Jugendlichen bedeutsam (Owens, 2017). Vernetzung und Selbstdarstellung erhöhen zudem die Sichtbarkeit marginalisierter Lebensformen und Gruppen. Digitale Medien haben dahingehend ein gesellschaftliches Empowerment- und Entwicklungspotenzial. Inwieweit dieses jedoch im Alltag einzelner und ganzer Gruppierungen Potenziale entfaltet, steht im Zusammenhang mit weiteren Ressourcen und führt zurück zu der Vorstellung einer Strukturverstärkerschaft. Ableismus - also die Benachteiligung von Menschen mit Behinderung -, Heteronormativität, Sexismus und gruppenbezogene Menschenfeindlichkeit sind Probleme des Online-Offline-Kontinuums. Denn digitale Medien erweitern auch Verdeckungszusammenhänge. Schließlich bleiben sowohl die Situation als auch die Betroffenheit ganzer Gruppen durch sich ergänzende Diskriminierungszusammenhänge unbeachtet (Bitzan et al., 2000). Letzteres äußert sich schon darin, dass sich junge Menschen gezwungen sehen, digitale Medien heimlich zu nutzen, wodurch sie nicht die Möglichkeit haben, sich mit Vertrauenspersonen vorurteilsfrei auszutauschen. Problematisch ist auch, wenn ihnen Reflexionsräume zur Auseinandersetzung mit medialen (Geschlechter-)Rollenbildern fehlen oder Kinder und Jugendliche mittels digitaler Medien versuchen, Bedürfnisse zu befriedigen, die aus dem sozialen Nahraum, der Familie, dem Freundeskreis oder besuchten Einrichtungen ansonsten ausgegrenzt werden. Mediatisierte Verdeckungszusammenhänge erhöhen demzufolge die Vulnerabilität von Kindern und Jugendlichen gegenüber mediatisierter sexualisierter Gewalt. Sie vereinfachen es Täter*innen, missbräuchliche Vertrauens- und Abhängigkeitsverhältnisse aufzubauen, indem sie Betroffenen sonst versagte Teilhabe suggerieren. Verdeckungszusammenhänge verstärken also die Folgebelastungen Betroffener. Sofern Letztere weitere Ausgrenzung befürchten, ist es Gewaltausübenden möglich, mit Schweigegeboten und Drohstrategien an ohnehin belastende Tabuisierungserfahrungen anzuknüpfen. Aus diesem Grund bleiben Betroffene oftmals ohne Hilfe. Teilhabebarrieren und Ausgrenzungserfahrungen gebührt deswegen im fachlichen Umgang mit mediatisierter sexualisierter Gewalt besondere Sensibilität. 


\section{Literatur}

Alencar, A., Kondova, K., \& Ribbens, W. (2019). The smartphone as a lifeline: An exploration of refugees' use of mobile communication technologies during their flight. Media, Culture \& Society, 41(6), 828-844. https://doi.org/10.1177/0163443718813486.

Bitzan, M., Funk, H., \& Stauber, B. (2000). Den Wechsel im Blick: Methodologische Ansichten feministischer Sozialforschung (2. Aufl.). Centaurus Verlag \& Media.

Broome, L. J., Izura, C., \& Davies, J. (2018). Linguistic characteristics of online grooming ,Relationships'. University of Swansea.

Connell, R. (2015). Der gemachte Mann. Springer VS.

Dekker, A., Koops, T., \& Briken, P. (2016). Sexualisierte Grenzverletzungen und Gewalt mittels digitaler Medien: Zur Bedeutung digitaler Medien für Phänomene sexualisierter Grenzverletzungen und Gewalt gegen Kinder und Jugendliche. Arbeitsstab des Unabhängigen Beauftragten für Fragen des sexuellen Kindesmissbrauchs.

Dekker, A., \& Koops, T. (2017). Sexting als Risiko? Zum konsensuellen und nichtkonsensuellen Versand persönlicher erotischer Fotos mittels digitaler Medien. Bundesgesundheitsblatt, Gesundheitsforschung, Gesundheitsschutz, 60(9), 1034-1039. https://doi.org/ 10.1007/s00103-017-2595-9.

Dollinger, B., \& Raithel, J. (2006). Einführung in Theorien abweichenden Verhaltens: Perspektiven, Erklärungen und Interventionen. Beltz Juventa.

Döring, N. (2019). Jugendsexualität heute. Zwischen Offline- und Online-Welten. In H.-J. Voß \& M. Katzer (Hrsg.), Geschlechtliche und sexuelle Selbstbestimmung durch Kunst und Medien: Neue Zugänge zur sexuellen Bildung (S. 219-244). Psychosozial Verlag.

Gámez-Guadix, M., \& Mateos-Pérez, E. (2019). Longitudinal and reciprocal relationships between sexting, online sexual solicitations, and cyberbullying among minors. Сотриters in Human Behavior, 94, 70-76. https://doi.org/10.1016/j.chb.2019.01.004.

Goh, J. X., Bandt-Law, B., Cheek, N. N., Sinclair, S., \& Kaiser, C. R. (2021). Narrow prototypes and neglected victims: Understanding perceptions of sexual harassment. Journal of Personality and Social Psychology. doi: https://doi.org/10.1037/pspi0000260.

Hall, M. (2018). Disability, discourse and desire: Analyzing online talk by people with disabilities. Sexualities, 21(3), 379-392. https://doi.org/10.1177/1363460716688675.

Hamby, S. (2017). On defining violence, and why it matters. Psychology of Violence, 7(2), 167-180. https://doi.org/10.1037/vio0000117.

Kärgel, K., \& Vobbe, F. (2020). Mediatisierte Gewalt - Diffusion - Transzendenz: Erscheinungsformen und Herausforderungen sexualisierter Gewalt mit digitalem Medieneinsatz. Kindesmisshandlung und -vernachlässigung, 23(1), 30-43.

Machimbarrena, J. M., Calvete, E., Ferández-Gonzálet, L., Álvarez-Bardón, A., ÁlvarezFernández, L., \& González-Cabrera, J. (2018). Internet risks: An overview of victimitzation in cyberbullying, cyber dating abuse, sexting, online grooming and problematic internet use. International Journal of Environmental Research and Public Health, 15(2471), $1-16$.

Matthiesen, S., \& Dekker, A. (2018). Jugendsexualität: Sexuelle Sozialisation im Zeitalter des Internets. In A. Lange, H. Reiter, S. Schutter, \& C. Steiner (Hrsg.), Handbuch Kindheits- und Jugendsoziologie: Mit 28 Abbildungen und 12 Tabellen (S. 379-392). Springer VS. 
Owens, Z. D. (2017). Is it facebook official? Coming out and passing strategies of young adult gay men on social media. Journal of Homosexuality, 64(4), 431-449. https://doi. org/10.1080/00918369.2016.1194112.

Rice, C., \& Watson, E. (2016). Girls and sexting: The missing story of sexual subjectivity in a sexualized and digitally mediated world. In J. Coffey, S. Budgeon, \& H. Cahill (Hrsg.), Learning bodies: The body in youth and childhood studies (S. 141-156). Springer International Publishing.

Valkenburg, P. M., Peter, J., \& Schouten, A. P. (2006). Friend networking sites and their relationship to adolescents' well-being and social self-esteem. Cyberpsychology \& Behavior: The Impact of the Internet, Multimedia and Virtual Reality on Behavior and Society, 9(5), 584-590. https://doi.org/10.1089/cpb.2006.9.584.

Vobbe, F., \& Kärgel, K. (2020). Sexualisierte Gewalt mit digitalem Medieneinsatz gegen Jungen: Geschlechterbezogene Risiken und Herausforderungen für die Prävention. Theorie und Praxis der Sozialen Arbeit, 1, 49-56.

Vobbe, F., \& Kärgel, K. (Im Druck). Hedonistic Utilitarianism: The strategic use of digital media along the online-offline continuum of sexualised violence. In L. Kuhle \& D. Stelzmann (Hrsg.), Sexual online grooming of children: Challenges for science and practice. Nomos. (Erscheint voraussichtlich im Oktober 2021).

Wagenknecht, P. (2007). Was ist Heteronormativität? Zu Geschichte und Gehalt des Begriffs. In J. Hartmann, C. Klesse, P. Wagenknecht, B. Fritzsche, \& K. Hackmann (Hrsg.), Heteronormativität (S. 17-34). Springer VS.

Wells, M., \& Mitchell, K. J. (2007). Youth sexual exploitation on the internet: DSM-IV diagnoses and gender differences in co-occurring mental health issues. Child and Adolescent Social Work Journal, 24(3), 235-260. https://doi.org/10.1007/s10560-007-0083-z.

Whittle, H. C., Hamilton-Giachritsis, C. E., \& Beech A. R. (2014). „Under His Spell“: Victims' perspectives of being groomed online. Social Sciences, 3, 404-426.

Open Access Dieses Kapitel wird unter der Creative Commons Namensnennung 4.0 International Lizenz (http://creativecommons.org/licenses/by/4.0/deed.de) veröffentlicht, welche die Nutzung, Vervielfältigung, Bearbeitung, Verbreitung und Wiedergabe in jeglichem Medium und Format erlaubt, sofern Sie den/die ursprünglichen Autor(en) und die Quelle ordnungsgemäß nennen, einen Link zur Creative Commons Lizenz beifügen und angeben, ob Änderungen vorgenommen wurden.

Die in diesem Kapitel enthaltenen Bilder und sonstiges Drittmaterial unterliegen ebenfalls der genannten Creative Commons Lizenz, sofern sich aus der Abbildungslegende nichts anderes ergibt. Sofern das betreffende Material nicht unter der genannten Creative Commons Lizenz steht und die betreffende Handlung nicht nach gesetzlichen Vorschriften erlaubt ist, ist für die oben aufgeführten Weiterverwendungen des Materials die Einwilligung des jeweiligen Rechteinhabers einzuholen.

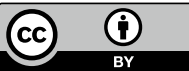

